\title{
Stimulus control of matching and oddity in a pigeon'
}

JOHN A. NEVIN AND KLAUS LIEBOLD

SWARTHMORE COLLEGE

A pigeon was trained on matching and oddity with red and green key colors. Illumination of a light served as a cue for matching or oddity. Injections of sodium pentobarbital and transfer to novel key colors affected matching and oddity differently, suggesting that stimulus control of two different kinds of performance had been established.

Pigeons are readily trained to peck a key of a hue which matches that of a standard stimulus, or to peck a key of different hue, by arranging the appropriate contingencies of reinforcement. Skinner (1950) noted that such matching and oddity performances appeared quite similar. However, subsequent data suggest that there may be some important differences. In particular, Cumming \& Berryman (1965) found differences in presolution behavior and in rate of acquisition of matching and oddity, and also found that oddity performance was less affected than matching by injections of sodium pentobarbital. Their comparisons were based on independent groups of pigeons. We have employed a successive discrimination technique to study both matching and oddity performances in an individual bird within single sessions. Briefly, we programmed the oddity contingency in the presence of a yellow light, and the matching contingency in its absence, using the same key colors (red and green) for both matching and oddity. In this procedure, the yellow light may be thought of as exercising control over a set of conditional discrimination performances, which may themselves involve instructional control by the standard stimulus (Cumming \& Berryman, 1965). As such, this procedure represents an interesting extension of the principles of stimulus control over the behavior of lower organisms.

Method

The $S$ was a male white Carneaux pigeon with an experimental history on multiple schedules of reinforcement in the presence of red and green lights. It was maintained at $85 \%$ of its free-feeding weight, about $440 \mathrm{gm}$. The bird was trained in a standard pigeon chamber with three keys, mounted 4 in. apart on centers, and with a grain magazine below the center key. A yellow pilot light was mounted 2 in. above the center key.

A trial began when the center key was illuminated with red or green light. When the bird pecked the center key, the side keys were illuminated, one red and one green, with the center key remaining on. The color of the center key, and the location of red or green on the sides, was predetermined by a random sequence with the restrictions that the four possible color combinations occurred equally often in blocks of 24 trials, and no more than three identical combinations occurred in succession. The sequence was changed several times during training. The bird was given 3 sec. access to grain for pecking the side key of matching hue when the yellow pilot light was off, and for pecking the non-matching side key when the yellow light was on. Pecks at the incorrect side key were followed by a 3-sec. blackout. In either event, a side key peck turned off all key lights for $10 \mathrm{sec}$. and advanced the trial sequence. Matching and oddity contingencies, cued by absence or presence of yellow light, alternated in blocks of 25 trials. Sessions started at a randomly chosen position in the trial sequence.

The bird was first trained to a high level of accuracy on the matching problem alone, in the absence of yellow light. The full procedure was then introduced. Sessions consisted of 150 trials and were run every other day. Fifty such sessions were required to establish high stable accuracies in both matching and oddity.

Alternate sessions were then given with and without sodium pentobarbital. When given, the drug was injected into the pectoral muscle $10 \mathrm{~min}$. before the start of the session. Doses of $2,3,4,5$, and $6 \mathrm{mg}$ were given in ascending order, with several repetitions of each dose except $2 \mathrm{mg}$.

At this point, the bird's beak was broken, and it was given several months of free feeding while the beak grew back. Its weight was then reduced to 440 gm again, and further training was given. After high accuracies in matching and oddity were re-established, the red and green key lights were replaced with yellow and blue. Ten sessions of 150 trials on the new colors were run with matching and oddity, cued by the yellow pilot light, alternating as before.

Results; drug test

At the end of original training, the bird was performing consistently between 86 and 99 per cent correct on both matching and oddity. Accuracies on both components of the procedure tended to decrease with increasing doses of pentobarbital, but the decrease in accuracy of matching was greater. This differentiation is indicated in Fig. 1, which plots accuracy of oddity as a function of accuracy of matching for both drug and control sessions. Control accuracies are about the same for both performances, clustering in the range from 86 to 100 percent correct. However, with a single exception, accuracies under drug are higher for oddity than for matching, indicated by the fact that the data points appear above the $45^{\circ}$ locus of equal accuracy. This replicates the 


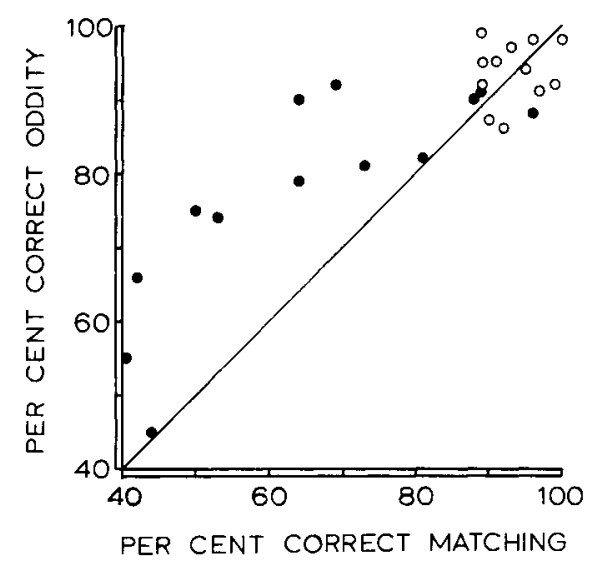

Fig. 1. Accuracy of oddity performance plotted against accuracy of matching for individual sessions with and without injections of sodium pentobarbital. Drug sessions are represented by the filled points. The $45^{\circ}$ line represents the locus of equal accuracy.

finding of Cumming \& Berryman (1965).

\section{Results: transfer to novel hues}

At the end of five sessions of retraining with red and green, the bird was performing at better than $95 \%$ correct in both matching and oddity. In the first session with yellow and blue, the bird chose the yellow side key about $80 \%$ of the time when yellow was on the center in the matching condition, and when blue was on the center in oddity (see Fig. 2). With blue on the center in matching, or with yellow on the center in oddity, the bird performed at chance, with no position preference. In brief, the bird exhibited a preference for yellow which was conditional upon the center key color and the cue for matching or oddity. Thus, there was at least partial transfer of both matching and oddity performances to novel key colors.

Changes in behavior over the course of the 10 transfer sessions were complex. It should be noted that accuracy of blue matching rose from chance to $100 \%$ correct, while the oddity performance with blue on the center fell to chance. It is clear that the illumination of the pilot light above the keys exercised control over different performances in the presence of the same key colors. The fact that blue matching came into conformity with the reinforcement contingency, while oddity did not despite its initial advantage in transfer, is similar to the observations of Cumming \& Berryman (1965) on acquisition of matching and oddity.

\section{Discussion}

In their analysis of transfer of oddity in pigeons, Cumming \& Berryman (1965) concluded that oddity involved learning to choose a correct, non-matching side key, rather than to avoid the key of matching hue. This

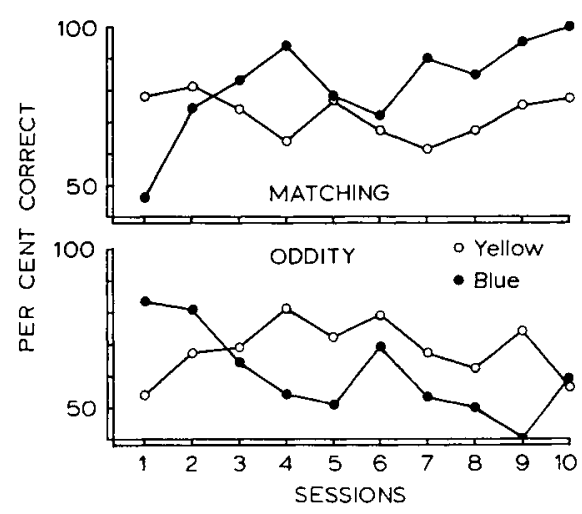

Fig. 2. Accuracy of matching and oddity performances with yellow or blue on the center key during 10 sessions of exposure to novel key colors.

was interpreted to mean that, in experiments with three key colors, birds had to learn twice as many stimulusresponse relations in oddity as in matching. For instance, in oddity with red on the center, they had to learn to peck blue or green, while in matching with red on the center, they only had to learn to peck red. Our study appears to have replicated the major differences between matching and oddity in their work, even though only two key colors were used, giving an equal number of stimulus-response relations to be learned in both matching and oddity. Thus, we are inclined to conclude that matching and oddity involve qualitatively different performances, even though they a re both conditional discriminations in which the standard stimulus acts to specify the correct side key.

The establishment of stimulus control over performances which can only be specified in terms of relations between stimuli raises the question of whether such performances are functionally similar to simple discrete operants. The fact that control was established in a pigeon within a reasonable period of training suggests that laboratory analysis of such complex operant discriminations is feasible.

\section{References}

Cumming, W. W., \& Berryman, R. The complex discriminated operant: studies of matching-to-sample and related problems. In D. Mostofsky (Ed.), Stimulus generalization. Stanford: Stanford University Press, 1965.

Skinner, B. F. Are theories of learning necessary? Psychol. Rev., $1950,57,193-216$.

\section{Note}

1. This research was supported by Grant MH-08515-02 from the National Institute of Mental Health to Swarthmore College. 